\title{
Successful Conservative Treatment of a Complicated Aortic Thrombus in a Woman with Factor V Leiden Mutation
}

\author{
Elise Frebutte ${ }^{1}$, Myriam Bibombe ${ }^{1}$, Arthur Dumont ${ }^{1}$, Maxime Haxhe ${ }^{2}$, Pascal Reper $^{1,3,4,5}$ \\ ${ }^{1}$ Internal Medicine Department, CHR Haute Senne, Soignies, Belgium \\ ${ }^{2}$ Radiology Department, CHR Haute Senne, Soignies, Belgium \\ ${ }^{3}$ Critical Care Department, CHR Haute Senne, Soignies, Belgium \\ ${ }^{4}$ Critical Care Department, CHU UCL Namur, Yvoir, Belgium \\ ${ }^{5}$ Federal Health Ministry, Brussels, Belgium
}

Doi: 10.12890/2021_002641 - European Journal of Case Reports in Internal Medicine - ๑ EFIM 2021

Received: $15 / 05 / 2021$

Accepted: $20 / 05 / 2021$

Published: 07/06/2021

\begin{abstract}
How to cite this article: Frebutte E, Bimbombe M, Dumont A, Haxhe M, Reper P. Successful conservative treatment of a complicated aortic thrombus in a woman with Factor V Leiden mutation. EJCRIM 2021;8: doi:10.12890/2021_002641.
\end{abstract}

Conflicts of Interests: The authors declare there are no competing interests.

This article is licensed under a Commons Attribution Non-Commercial 4.0 License

\section{ABSTRACT}

Thrombus in the aortic trunk is a rare complication. We report the case of a 63-year-old patient with a factor $\mathrm{V}$ Leiden mutation in whom an aortic arch thrombus was discovered accidentally. Conservative treatment was initiated with therapeutic anticoagulation with lowmolecular-weight heparin leading to complete thrombus lysis after 3 months but associated shortly after anticoagulation initiation with a large splenic and limited renal infarctions.

\section{LEARNING POINTS}

- Intra-aortic thrombus is rarely diagnosed on routine CT examination.

- Even a complicated aortic arch thrombus can be successfully treated with conservative anticoagulation.

- Vascular systemic embolisms are possible after therapeutic anticoagulation is started.

\section{KEYWORDS}

Aortic thrombus, factor $\mathrm{V}$ Leiden, anticoagulation, embolism

\section{CASE DESCRIPTION}

A 63-year-old woman was admitted in the emergency department with dyspnoea and cough. Her medical history included pulmonary embolisms related to a heterozygous factor $V$ Leiden mutation. She was not on long-term anticoagulation.

Arterial blood gas analysis on admission showed severe hypoxaemia ( $\mathrm{PaO}_{2} 47 \mathrm{mmHg}$ without oxygen) related to pulmonary infiltrates on chest $x$-ray treated with antibiotic therapy with a combination of amoxicillin/clavulanate acid and clarithromycin (Fig. 1).

To exclude a new pulmonary embolism secondary to the coagulation disorders associated with factor $\mathrm{V}$ mutation, thoracic CT angiography was performed showing no pulmonary embolisms but revealing a large thrombus at the aortic arch junction with the descending aorta, measuring $9 \mathrm{~mm}$ in thickness and extending for $5 \mathrm{~cm}$. The most distal part of this thrombus seemed to be detached from the intima-media complex (Fig. 2).

While the patient's respiratory situation progressively improved 2 days after anticoagulation was started, she developed acute pain in her left upper abdomen. An abdominal CT scan was immediately performed showing a large splenic infarction associated with a possible right 


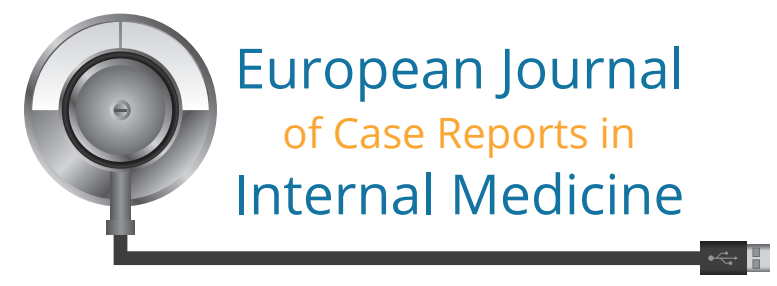

renal infarction (Fig. 3). It was decided to continue the conservative therapeutic anticoagulation without surgery. A CT scan performed 3 months later showed complete resolution of the aortic arch thrombus without any new embolic complications or clinical problems.

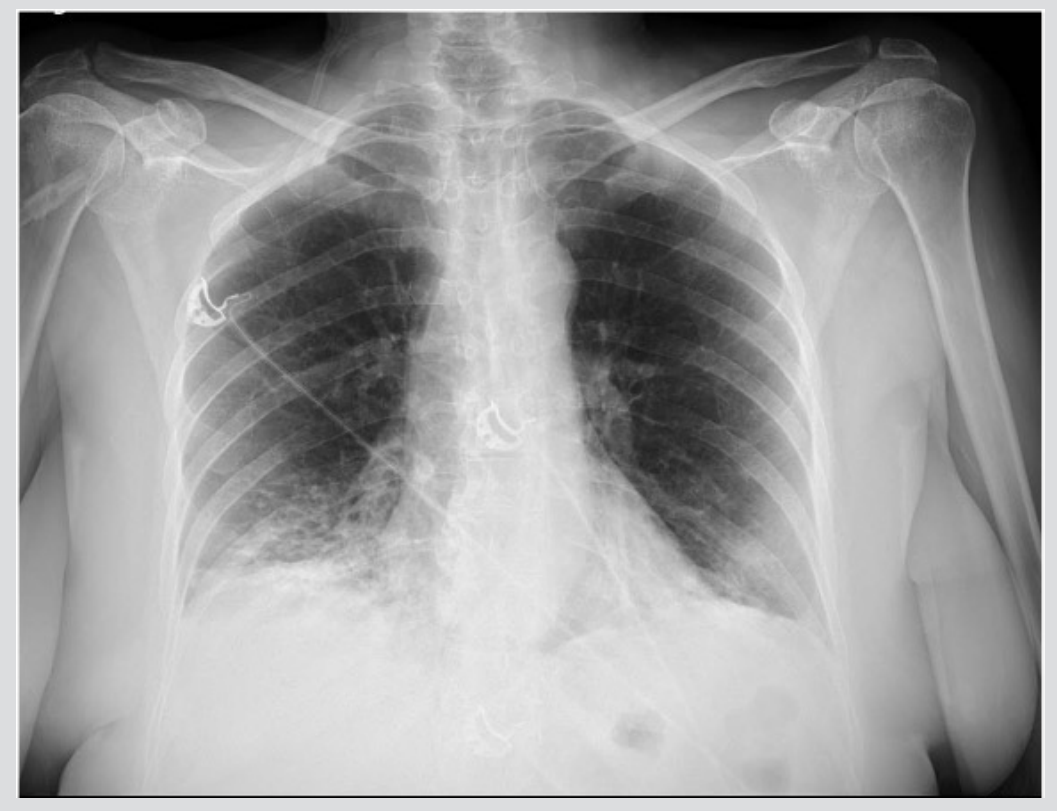

Figure 1. Chest $x$-ray on admission showing bibasal infiltrates (arrow)
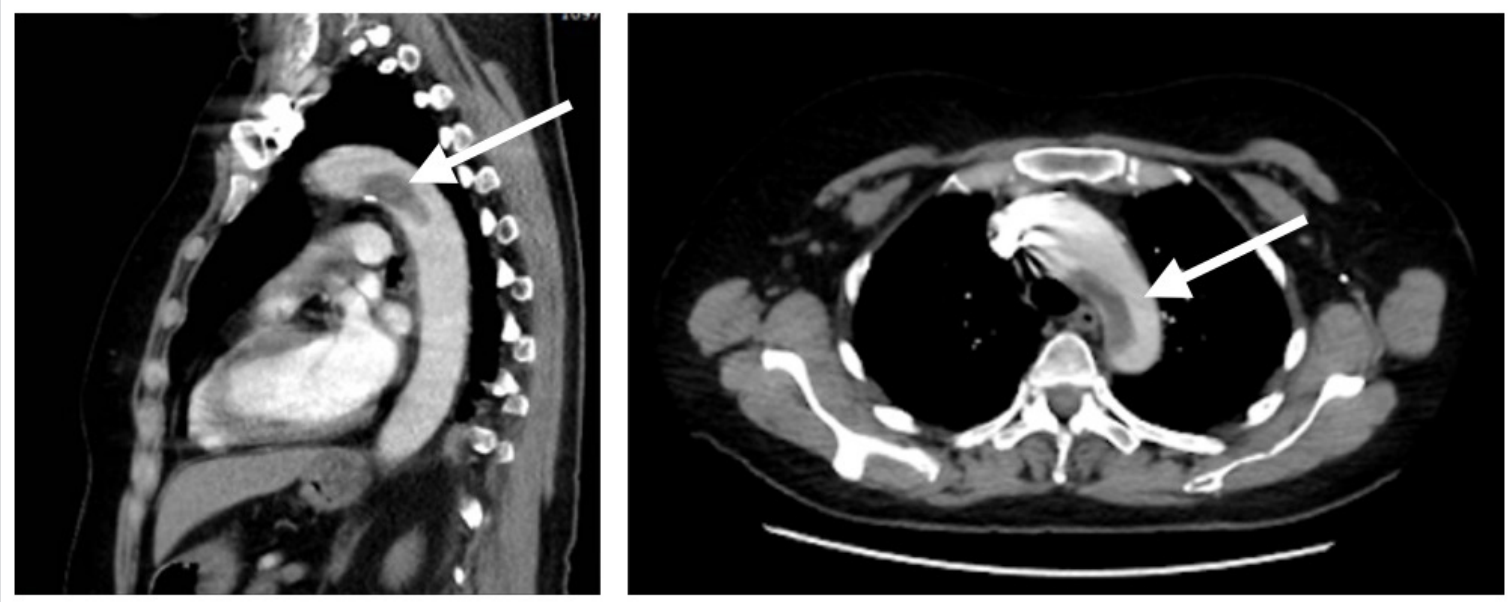

Figure 2. Thoracic CT angiogram on admission showing a thick thrombus occurring at the junction of the aortic arch and descending thoracic aorta (arrows)
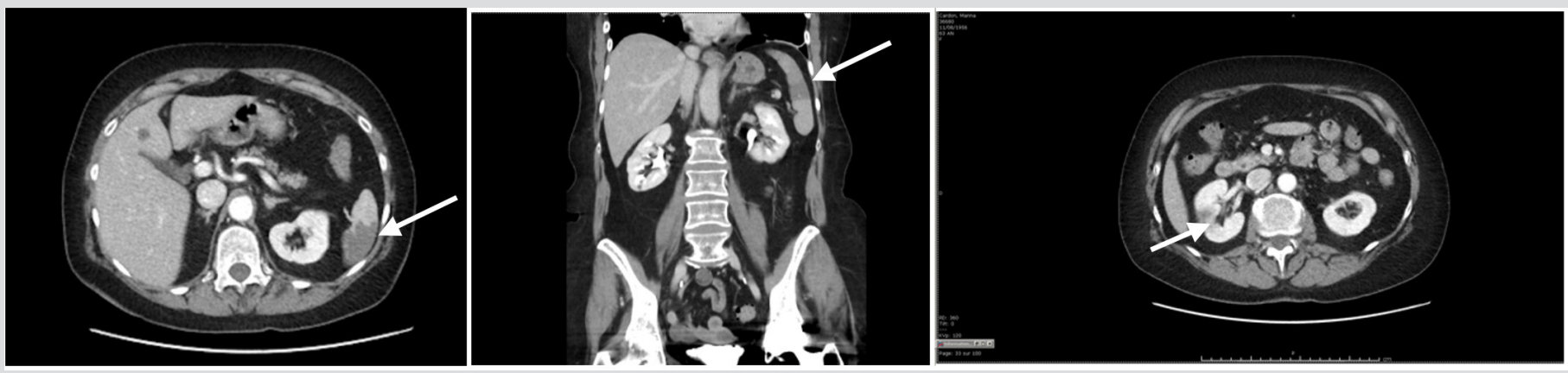

Figure 3. Abdominal CT scan showing a large splenic infarction with a hypodense zone in the middle part of the right kidney related to a renal infarction (arrows) 


\section{DISCUSSION}

Asymptomatic thrombus in the aortic arch in the absence of underlying atherosclerosis or aortic aneurysm is very rare. Fragmentation is often responsible for acute ischaemic episodes caused by embolisms in various locations like the lower and upper limbs, but also including visceral (renal, splenic, intestinal) and cerebral locations ${ }^{[1,2]}$.

Aortic thrombi are frequently secondary to coagulation disorders, such as the factor $\mathrm{V}$ mutation in our patient, but protein $\mathrm{C}$ and $\mathrm{S}$ deficiency, homocysteinaemia, elevated factor VIII levels, antithrombin III deficiency, antiphospholipid antibody syndrome, polycythaemia vera, systemic lupus erythematosus, infectious disease, malignancy, trauma and instrumentation can also be responsible for aortic thrombus development ${ }^{[3]}$.

Although most cases of aortic thrombus are diagnosed after embolic events have occurred, some can found incidentally during routine examination, as in our patient: in this case, the embolic complications were probably secondary to the initiation of anticoagulation leading to progressive lysis of the thrombus. Systemic consequences were limited to acute abdominal pain without functional implications related to the splenic and renal embolisms.

The therapeutic approach is still debated and the ideal treatment for such lesions is poorly defined. Treatments should focus on preventing extension of these lesions potentially leading to multiple embolic complications. A variety of approaches are used including thrombolysis, interventional modalities such as thromboaspiration or balloon-catheter thrombectomy and open surgical procedures with thrombectomy, thromboendarterectomy or aortic prosthetic arch replacement ${ }^{[4-6]}$. Clinical experience remains important in deciding on the therapeutic approach in these uncommon situations, with conservative treatment with anticoagulation remaining another option ${ }^{[7-9]}$.

Most of the published cases reported that aortic thrombi are discovered after an initial thrombotic event leading to the diagnosis. In our case, renal and splenic embolisms probably resulted from the therapeutic anticoagulation for the large aortic arch thrombus diagnosed during a routine $\mathrm{CT}$ scan to exclude a new pulmonary embolism in a patient with factor $\mathrm{V}$ mutation.

This observation suggests that aortic thrombus could be detected before clinical thrombotic manifestations and confirms that even with a large aortic arch thrombus and embolic systemic complications, a conservative approach with therapeutic anticoagulation can lead to complete resolution of this serious complication of well-known coagulation disorders.

\section{REFERENCES}

1. Li HD, Sun TC. Bowel ischemia caused by a giant thrombus in the ascending aorta. A case report. Vascular 2015;23:641-644.

2. Tsilimparis N, Hanack U, Pisimisis G. Thrombus in the non-aneurysmal, non-atherosclerotic descending thoracic aorta: an unusual source of arterial embolism. Eur J Vasc Endovasc Surg 2011;41:450-457.

3. Choukroun EM, Labrousse LM, Madonna FP. Mobile thrombus of the thoracic aorta: diagnosis and treatment in 9 cases. Ann Vasc Surg 2002;16:714-722.

4. Pagni S, Trivedi J, Ganzel BL. Thoracic aortic mobile thrombus: is there a role for early surgical intervention? Ann Thorac Surg 2011;91:1875-1881.

5. Deschka H, Erler S, Machner M. Surgery of the ascending aorta, root remodelling and aortic arch surgery with circulatory arrest through partial upper sternotomy: results of 50 consecutive cases. Eur J Cardiothorac Surg 2013;43:580-584.

6. Roselli EE, Idrees J, Greenberg RK. Endovascular stent grafting for ascending aorta repair in high-risk patients. J Thorac Cardiovasc Surg 2015;149:144-151.

7. Mahnken AH, Hoffman A, Autschbach R. Bare metal stenting for endovascular exclusion of aortic arch thrombi. Cardiovasc Intervent Radiol 2013;36:1127-1131.

8. Rancic Z, Pfammatter T, Lachat M. Floating aortic arch thrombus involving the supraaortic trunks: successful treatment with supra-aortic debranching and antegrade endograft implantation. J Vasc Surg 2009;50:1177-1180.

9. Melissano G, Tshomba Y, Bertoglio L. Analysis of stroke after TEVAR involving the aortic arch. Eur J Vasc Endovasc Surg 2012;43:269-275. 